\title{
Noninvasive photoacoustic computed tomography of mouse brain metabolism in vivo
}

\author{
Junjie Yao ${ }^{1, \uparrow}$, Jun Xia ${ }^{1, \Upsilon}$, Konstantin I. Maslov ${ }^{1}$, Mohammadreza Nasiriavanaki ${ }^{1}$, Vassiliy \\ Tsytsarev $^{2}$, Alexei V. Demchenko ${ }^{3}$, and Lihong V. Wang ${ }^{1, *}$ \\ ${ }^{1}$ Optical Imaging Laboratory, Department of Biomedical Engineering, Washington University in St. \\ Louis, St. Louis, MO 63130, USA \\ ${ }^{2}$ Department of Anatomy and Neurobiology, University of Maryland School of Medicine, \\ Baltimore, MD 21201, USA \\ ${ }^{3}$ Department of Chemistry and Biochemistry, University of Missouri-St. Louis, St. Louis, MO \\ 63121, USA
}

\begin{abstract}
We have demonstrated the feasibility of imaging mouse brain metabolism using photoacoustic computed tomography (PACT), a fast, noninvasive and functional imaging modality with optical contrast and acoustic resolution. Brain responses to forepaw stimulations were imaged transdermally and transcranially. 2-NBDG, which diffuses well across the blood-brain-barrier, provided exogenous contrast for photoacoustic imaging of glucose response. Concurrently, hemoglobin provided endogenous contrast for photoacoustic imaging of hemodynamic response. Glucose and hemodynamic responses were quantitatively decoupled by using two-wavelength measurements. We found that glucose uptake and blood perfusion around the somatosensory region of the contralateral hemisphere were both increased by stimulations, indicating elevated neuron activity. While the glucose response area was more homogenous and confined within the somatosensory region, the hemodynamic response area had a clear vascular pattern and spread wider than the somatosensory region. Our results demonstrate that 2-NBDG-enhanced PACT is a promising tool for noninvasive studies of brain metabolism.
\end{abstract}

\section{Keywords}

photoacoustic computed tomography; brain imaging; 2-NBDG; forepaw stimulation; glucose metabolism; hemodynamic response

\section{Introduction}

In mammals, the brain coherently controls the overall action of the body (Carew, 2000). Performing numerous computation-intensive tasks such as information processing, perception, motor control and learning, brain tissue consumes a large amount of energy in proportion to its volume (Nieuwenhuys et al., 1998). For example, humans devote 20-25\% of their metabolism to the brain, where the energy is mostly used for sustaining the electric

\footnotetext{
*Corresponding author: LHWANG@WUSTL.EDU Address: One Brookings Drive, Campus Box1097, Washington University in St. Louis, St. Louis, MO 63130 Phone: (314) 935-6152 Fax: (314) 935-7448.

IlThese authors contributed equally to this work

Lihong Wang has a financial interest in Endra, Inc., which, however, did not support this work. Lihong Wang and K. Maslov have a financial interest in Microphotoacoustics, Inc., which did not support this work.
} 
charge (membrane potential) of neurons (Mink et al., 1981; Nieuwenhuys et al., 1998). In humans and many other species, the brain gets most of its energy from oxygen-dependent metabolism of glucose (Nieuwenhuys et al., 1998). An abnormal metabolic rate of glucose and/or oxygen usually reflects a diseased status of brain, such as cancer or Alzheimer's disease (Fulham et al., 1992; Mosconi et al., 2009). In addition, physiologically active regions of the cerebral cortex consume more energy than inactive regions (Raichle and Gusnard, 2002). These phenomena have formed the basis for functional brain imaging methods, including positron emission tomography (PET) and functional magnetic resonance imaging (fMRI) (Mehagnoul-Schipper et al., 2002; Schlemmer et al., 2008).

However, PET depends on the administration of radioactively-labeled tracers (e.g., 2deoxy-2-fluoro-D-glucose, FDG), a complex procedure with exposure to ionizing radiation. fMRI is primarily sensitive to deoxy-hemoglobin and suffers from slow imaging speed. Moreover, both PET and fMRI are expensive techniques with poor spatial resolutions. Therefore, a fast, noninvasive and non-ionizing imaging modality with good spatial resolutions is needed to advance metabolism-associated studies of brain physiology and pathology.

On the basis of the photoacoustic effect, photoacoustic tomography (PAT) solves the resolution drawback of pure optical imaging and the contrast drawback of pure ultrasonic imaging (Itoh et al., 2004; Wang, 2008, 2009a; Yao and Wang, 2011). In PAT, photon energy absorbed by molecules is partially or completely converted into heat, which thermoelastically induces pressure waves. The induced pressure waves are detected by ultrasonic detectors to form an image (Itoh et al., 2004). PAT is capable of anatomical, functional, molecular and metabolic imaging of small animals, with highly scalable spatial resolution and penetration depth (Wang, 2009a; Wang, 2009b; Yao and Wang, 2011; Zhang et al., 2006). Photoacoustic computed tomography (PACT) is a major implementation of PAT, which aims at fast data acquisition, sub-millimeter resolution and deep penetration depth beyond the optical diffusion limit (Li et al., 2010; Li et al., 2008; Wang et al., 2003; Xia et al., 2011). Noninvasive, label-free and functional PACT of the rat brain was demonstrated by accurately mapping brain lesions and cerebral hemodynamics (Wang et al., 2003). Molecular imaging of a mouse brain tumor in vivo was also performed with PACT, using IRDye-800-c as the contrast agent (Li et al., 2008).

Here, for the first time, we have demonstrated that PACT is able to image glucose uptake in the mouse brain, using a newly developed glucose analog 2-deoxy-2-[N-(7-nitrobenz-2oxa-1,3-diazol-4-yl)amino]-D-glucopyranose (2-NBDG). To demonstrate the metabolic imaging capability of PACT, we studied in vivo forepaw stimulation responses. Our phantom and animal studies showed that PACT could spectrally separate 2-NBDG and blood using two-wavelength measurements, thus decouple the glucose and hemodynamic responses to the stimulations. Open-scalp photoacoustic microscopy and fluorescence imaging were used to validate the results from PACT.

\section{Materials and methods}

\subsection{2-NBDG}

2-NBDG is a newly developed fluorescent 2-deoxyglucose (2-DG) analog (Bem et al., 2007; Cheng et al., 2006; Gaudreault et al., 2008; Itoh et al., 2004; Langsner et al., 2011; Millon et al., 2011; O'Neil et al., 2005; Sheth et al., 2009; Tsytsarev et al., 2012). Like the FDG (molecular weight: 181) used in PET studies, 2-NBDG is transported into cells via the same GLUT as glucose (Sheth et al., 2009). Once taken up by cells, 2-NBDG is phosphatized to 2-NBDG-6-P, which prevents it from being released again from the cells. However, because of the lack of the 2-hydroxyl group needed for glycolysis, 2-NBDG-6-P cannot be further 
metabolized. Therefore, the distribution of trapped 2-NBDG is a good reflection of glucose metabolism (O'Neil et al., 2005). Because 2-NBDG is a relatively small molecule (molecular weight: 342) [Figure 1a], it crosses the blood-brain-barrier much more easily than other near-infrared fluorophore-labeled 2-DG analogs, such as IRDye800-DG (molecular weight: 1330) (Cheng et al., 2006; Itoh et al., 2004). Moreover, 2-NBDG has its peak absorption at $478 \mathrm{~nm}$, where hemoglobin has a much lower absorption [Figure 1b]. By carefully controlling the laser fluence, the signal contribution from hemoglobin can be neglected at this wavelength. These features have made 2-NBDG particularly suitable for brain studies.

\subsection{Noninvasive photoacoustic computed tomography (PACT)}

Figure 2a is the schematic of the PACT setup. An OPO laser (BasiScan 120, SpectraPhysics) is pumped by a Nd:YAG laser (Brilliant B, Quantel) with a third harmonic generator $(355 \mathrm{~nm})$ to provide pulses with wavelengths tunable from $420 \mathrm{~nm}$ to $680 \mathrm{~nm}$. The pulse duration is $6 \mathrm{~ns}$, and the pulse repetition rate is $10 \mathrm{~Hz}$. The laser beam is homogenized by an optical diffuser (EDC-5, RPC Photonics) to provide uniform illumination over the mouse brain. The maximum light intensity at the tissue surface is approximately $10 \mathrm{~mJ} / \mathrm{cm}^{2}$, below the ANSI limit at the chosen wavelengths. The photoacoustic signals are detected by a $5 \mathrm{~cm}$ diameter full-ring ultrasonic transducer array with 512 elements (Imasonic, Inc.). The central frequency of the ultrasonic transducer array is $5 \mathrm{MHz}$, and the 6-dB bandwidth is more than $80 \%$. Each element in the array is directly shaped into an arc to produce an axial focal depth of $19 \mathrm{~mm}$. The combined foci of all elements form a relatively uniform imaging region of $20 \mathrm{~mm}$ diameter and $1 \mathrm{~mm}$ thickness. Within this region, the axial (radial) resolution is $0.10 \mathrm{~mm}$, and the transverse (tangential) resolution is $0.25 \mathrm{~mm}$ (Gamelin et al., 2009). As show in Figure 2b, after a complete data acquisition from all 512 elements, the raw data is reconstructed to form a photoacoustic image of the brain based on the universal back-projection algorithm (Xu and Wang, 2007). The imaging speed of the current system is $1.6 \mathrm{sec}$ per frame.

\subsection{Optical-resolution photoacoustic microscopy (OR-PAM)}

OR-PAM, another implementation of PAT, aims at capillary-level resolution within the optical diffusion limit (Hu et al., 2011; Maslov et al., 2008; Yao et al., 2009; Yao et al., 2010; Yao et al., 2011; Yao and Wang, 2010, 2011). As shown in Figure 2c, by focusing the laser pulses to a diffraction-limit spot using an objective with an NA of 0.1 (AC127-050-A, Thorlabs), OR-PAM achieves a transverse resolution of $5 \mu \mathrm{m}$. By using a single-element ultrasonic transducer with a central frequency of $50 \mathrm{MHz}$ and a 6-dB bandwidth of $100 \%$ (V214-BB-RM, Olympus-NDT), OR-PAM achieves an axial resolution of $15 \mu \mathrm{m}$. Due to scattering by the tissue, the penetration depth of OR-PAM is limited to $\sim \mathrm{mm}$, which is sufficient for transcranial imaging of the cerebral cortex but not transdermal imaging. The arrival time of the PA signal provides depth information, and volumetric imaging is acquired by two-dimensional raster scanning of the sample. In this study, to validate the cortical vascular image obtained by PACT, the same mouse was imaged by OR-PAM at $570 \mathrm{~nm}$ after the PACT imaging. The scalp was surgically removed, while the skull was left intact. It took about $30 \mathrm{~min}$ to acquire an OR-PAM image over a $5 \times 10 \mathrm{~mm}^{2}$ area.

\subsection{Fluorescence imaging}

To confirm the stimulation induced changes in 2-NBDG uptake in brain, open-scalp fluorescence imaging using the same experimental protocol was performed on a different mouse. The fluorescence imaging system shown in Figure $2 \mathrm{~d}$ has been reported previously (Tsytsarev et al., 2012). Briefly, the light source is a 120-watt xenon arc lamp (Oriel Inc.) with an excitation filter ( $450 \pm 20 \mathrm{~nm}, \mathrm{FB} 450-40$, Thorlabs). Fluorescence images are captured by a low-noise CCD camera (Meade Inc.). An emission filter $(550 \pm 20 \mathrm{~nm}$, 
FB550-40, Thorlabs) and an achromatic doublet (NT45-265, Edmund) comprise the camera lens. In this study, the fluorescence images were acquired with a CCD exposure time of 0.5 sec. White-light images were acquired with a CCD exposure time of $0.05 \mathrm{sec}$ after removing the emission filter and turning on the room light. Because blood vessels appeared darker than the background in the white-light images, the image intensity was reversed for better clarity.

\subsection{Animal preparation}

Female ND4 Swiss Webster mice (Harlan Laboratory, 16 to 20g) were used for the current study. The laboratory animal protocols for this work were approved by the Animal Studies Committee of Washington University in St. Louis. To enhance 2-NBDG uptake, the mice were fast for $24 \mathrm{~h}$ before the experiment (Sheth et al., 2009). Before imaging, the hair on each mouse's head was removed with a depilatory. An intraperitoneal dose of $90 \mathrm{mg} / \mathrm{kg}$ ketamine plus $10 \mathrm{mg} / \mathrm{kg}$ xylazine was used for anesthesia. The mouse was then taped to a lab-made animal holder, which was then mounted to the PACT system. Instead of being fully immersed in water, the animal was supported from below, with the head being covered by a flexible membrane. The entire experiment took less than one hour, therefore additional injection of the anesthetic mixture was not needed. The animal preparation for OR-PAM and fluorescence imaging were similar, except that there was no water coupling for fluorescence imaging. Three mice were used for PACT and subsequent OR-PAM imaging, and one mouse was used for fluorescence imaging.

\subsection{Forepaw stimulation}

Thirty minutes after the injection of $0.3 \mathrm{~mL} 3 \mathrm{mM}$ 2-NBDG via the tail vein, stimulations were introduced by two pairs of needle electrodes inserted under the skin of the right and left forepaws, respectively. The electrodes were connected to a function generator (DS345, Stanford Research Systems) through a manual switch. The whole procedure consisted of four periods, each lasting for 3 min [Figure 3a]. The first and third periods $\left(\mathrm{P}_{1}\right.$ and $\left.\mathrm{P}_{3}\right)$ were resting states, while the second period $\left(\mathrm{P}_{2}\right)$ was right paw stimulation (RPS) and the fourth period $\left(\mathrm{P}_{4}\right)$ was left paw stimulation (LPS). Each stimulation period consisted of a train of electrical pulses with an amplitude of $2 \mathrm{~mA}$, a pulse width of $0.25 \mathrm{sec}$ and a repetition rate of $2 \mathrm{~Hz}$ [Figure 3b]. PACT acquired images continuously through the four periods. The procedure was first performed for 12 minutes at $478 \mathrm{~nm}$ and then repeated for 12 minutes at $570 \mathrm{~nm}$ after a delay of $10 \mathrm{~min}$. We assumed that brain responses were repeatable within the time window of the experiment.

\subsection{Image co-registration}

Before quantitative analysis, the PA images at $570 \mathrm{~nm}$ from OR-PAM and PACT were coregistered by using a MATLAB Image Processing Toolbox (R2010b, Mathworks, Boston, MA). Here, a 2D rigid registration was performed, which included linear translation, scaling, and rotation. The first step was to manually select $10-20$ control points in the two images. The spatial translation, scaling and rotation matrix was computed based on the coordinates of these control points. The entire OR-PAM image was then transformed and registered to the PACT image using this matrix. To show the co-registration performance, the coregistered OR-PAM image was superimposed on top of the PACT image, with the overly transparency proportional to the OR-PAM image pixel values.

\subsection{Signal processing}

In PACT, for both phantom and in vivo studies, the images acquired at $478 \mathrm{~nm}$ and $570 \mathrm{~nm}$ were used to reconstruct the distribution of 2-NBDG and hemoglobin, respectively. Since deoxy- and oxy-hemoglobin have the same absorption coefficients at $570 \mathrm{~nm}$, the PA signal 
amplitude was proportional to the total hemoglobin concentration $C_{H b}$, an index for blood perfusion.

For open-scalp OR-PAM, because the mouse skull is translucent, the attenuation of light due to the skull was neglected. The laser fluence measured at the skull surface is a good approximation of the actual fluence at the blood vessels. Therefore, $C_{H b}$ can be estimated as (Wang and $\mathrm{Wu}, 2007$ )

$$
C_{H b}=\frac{V_{O R}^{570}}{k_{O R} \varepsilon_{H b}^{570} F_{O R}^{570}} .
$$

Here, the subscript $O R$ denotes the OR-PAM system and the superscript 570 denotes the wavelength. $k_{O R}$ is a calibration factor measured from blood in a clear medium, which incorporates the system detection efficiency and Grueneisen coefficient. $\varepsilon_{H b}^{570}$ is the molar extinction coefficient of hemoglobin at $570 \mathrm{~nm}, F_{O R}^{570}$ is the laser fluence at the skull surface, and $V_{O R}^{570}$ is the OR-PAM signal amplitude.

Because PACT shares the same absorption contrast as OR-PAM, the calibrated $C_{H b}$ from OR-PAM can be directly transferred to PACT once the images from the two systems are coregistered. We estimate the calibration factor $k_{C T}$ for PACT by

$$
k_{C T}=\frac{V_{C T}^{570}}{C_{H b} \varepsilon_{H b}^{570} F_{C T}^{570}} .
$$

Here, the subscript $C T$ denotes the PACT system. $F_{C T}^{570}$ is the laser fluence measured at the scalp surface and $V_{C T}^{570}$ is the PACT signal amplitude. In addition to the system detection efficiency and Grueneisen coefficient, $k_{C T}$ also incorporates an attenuation factor of the laser fluence at $570 \mathrm{~nm}$, which cannot be neglected in PACT owing to the scalp.

The fluence attenuation of the scalp is wavelength dependent. However, the effective attenuation coefficients of the nude mouse scalp at $478 \mathrm{~nm}\left(7.1 \mathrm{~cm}^{-1}\right)$ and $570 \mathrm{~nm}(6.5$ $\mathrm{cm}^{-1}$ ) are approximately the same (Vishwanath et al., 2009), and the mouse scalp is relatively thin (less than $1.5 \mathrm{~mm}$ ) (Azzi et al., 2005). Therefore, the fluence attenuations at the two wavelengths were treated as the same. We also assumed that the heterogeneity of the scalp was negligible. Accordingly, we can estimate the 2-NBDG concentration $C_{2-N B D G}$ as

$$
C_{2-\mathrm{NBDG}}=\frac{V_{C T}^{478}}{k_{C T} \varepsilon_{2-\mathrm{NBDG}}^{478} \eta_{2-\mathrm{NBDG}} F_{C T}^{478}}
$$

Here, $\varepsilon_{H b}^{570}$ is the molar extinction coefficient of $2-\mathrm{NBDG}$ at $478 \mathrm{~nm} . \eta_{2-\mathrm{NBDG}}$ is the conversion efficiency from absorbed optical energy to heat, which is $45 \%$ for 2-NBDG (Natarajan and Srienc, 1999). The conversion efficiency for hemoglobin is absent in Eq. 2 because it is $100 \%$.

Eqs. (1-3) can be used to estimate the absolute concentrations of hemoglobin and 2-NBDG in the brain. Because the absolute baseline signals vary across the brain, relative changes are more robust for quantifying the responses induced by stimulations. However, it is also important to demonstrate the absolute imaging capability of PACT, which is useful for the baseline mapping. In addition, the future quantification of the metabolic rates of oxygen and glucose requires the absolute concentrations. 
In forepaw stimulations, PACT images acquired during each period $\left(\mathrm{P}_{1-4}\right)$ were averaged at each wavelength to increase the signal-to-noise ratio. The relative changes were computed as $\left(\mathrm{P}_{2}-\mathrm{P}_{1}\right) / \mathrm{P}_{1}$ for RPS, and as $\left(\mathrm{P}_{4}-\mathrm{P}_{3}\right) / \mathrm{P}_{3}$ for LPS. Only pixels with amplitude above 1.5 times the noise level were used for calculation. In response quantifications, only pixels with response magnitude above $10 \%$ of the maximum magnitude were averaged. The same procedure was also applied to the fluorescence imaging results.

Response areas at the two wavelengths were also quantified. Here, the response area was defined by the pixels with a response magnitude above $10 \%$ of the maximum response magnitude. The cortical area covered by the responding vessels was estimated as well. An ellipse equation was used to fit the outline of the responding vessels, with the center, major axis, minor axis and orientation as the fitting variables. The area of the best fit ellipse was approximated as the covered area.

\section{Results}

\subsection{PACT of 2-NBDG and blood phantom}

A phantom study was first performed to validate the spectral separation of 2-NBDG and blood. As shown in Figure 4a, whole bovine blood (hemoglobin concentration: $2.5 \mathrm{mM}$ ) and $0.4 \mathrm{mM}$ 2-NBDG solution were embedded in gelatin and imaged by PACT at $478 \mathrm{~nm}$ and $570 \mathrm{~nm}$. This 2-NBDG concentration was close to the estimated 2-NBDG concentration in animal studies to be performed later. The ratios between the averaged PA signal amplitudes of 2-NBDG and blood at $478 \mathrm{~nm}$ and $570 \mathrm{~nm}$ were $9: 1$ and 0.06:1, respectively [Figure 4b]. Blood was almost invisible at $478 \mathrm{~nm}$, so was 2-NBDG at $570 \mathrm{~nm}$. The PA amplitude of the 2-NBDG sample happened to be nearly identical to (0.98 times) that of the blood sample at $570 \mathrm{~nm}$. Therefore, 2-NBDG and blood could be separated as shown in Figure 4c. In addition, we found that 2-NBDG was more diffusive than hemoglobin (molecular weight: $64,458)$ in gelatin, due to the much smaller size of 2-NBDG molecules.

\subsection{PACT of mouse brain}

Figures $4 \mathrm{e}-\mathrm{f}$ are noninvasive PACT images of a mouse brain, acquired 30 min after 2NBDG administration. At $478 \mathrm{~nm}$, the PA signal amplitudes reflected the 2-NBDG concentration in the brain tissue [Figure 4e]. At $570 \mathrm{~nm}$, the PA signal amplitudes reflected the total hemoglobin concentration in blood vessels [Figure 4f]. Cortical vascular landmarks including the sagittal sinus (SS) and coronal suture (CS) were clearly imaged by PACT and confirmed by open-scalp photography. A merged image of $\mathrm{C}_{\mathrm{Hb}}$ and $\mathrm{C} 2-\mathrm{NBDG}$ is shown in Figure 4g, where 2-NBDG signals from blood vessels were excluded. The hemoglobin distribution was superimposed on top of the 2-NBDG distribution, with the transparency of the hemoglobin layer proportional to its pixel values. Capillary-level OR-PAM images of the same mouse are shown in Figures 5a-b. The co-registration results show that the transdermal and transcranial PACT image agreed well with the open-scalp OR-PAM image, as shown in Figures 5c-d.

\subsection{PACT of cortical responses to forepaw stimulations}

Figures 6a-b are the relative changes of PA signals induced by forepaw stimulations. At 478 $\mathrm{nm}$ [Figure 6a], the RPS and LPS caused PA signal amplitudes to increase by $3.6 \% \pm 2.2 \%$ and $2.0 \% \pm 1.1 \%$ in the somatosensory region (SR) of the contralateral hemisphere, respectively. Such increases indicate elevated glucose uptake rates, and thus reflect increased neuron activity. Under the two stimulations, the response areas were $5.7 \pm 1.3$ $\mathrm{mm}^{2}$ and $7.4 \pm 2.5 \mathrm{~mm}^{2}$, respectively. 
Similarly, at $570 \mathrm{~nm}$ [Figure 6b], the RPS and LPS caused PA signal amplitudes to increase by $6.4 \% \pm 5.3 \%$ and $3.2 \% \pm 2.9 \%$ in the contralateral hemisphere, respectively. Such changes indicate an increase in total hemoglobin concentration arising from elevated inflows of fresh blood, and thus reflect increased neuron activity as well. Under the two stimulations, the response areas were $4.7 \pm 1.5 \mathrm{~mm}^{2}$ and $4.5 \pm 1.2 \mathrm{~mm}^{2}$, respectively. Furthermore, the responding vessels covered much larger cortical regions of $16.5 \pm 3.3 \mathrm{~mm}^{2}$ and $15.1 \pm 2.5 \mathrm{~mm}^{2}$, respectively, as shown in Figure 7a. The areas covered by the responding vessels were significantly wider than the 2-NBDG response areas for both RPS and LPS, with $p$ values less than 0.05 (Figure 7b).

In addition, the linear regression shows that the 2-NBDG response amplitude was approximately proportional to that of the hemoglobin response for both RPS and LPS, with ratios of $\sim 0.48$ and $\sim 0.52$, respectively (Figure 8 a). Similarly, the ratios between the $2-$ NBDG response area and the area covered by the responding vessels were $\sim 0.41$ and $\sim 0.57$ for RPS and LPS, respectively (Figure 8b). The high correlation of the two responses may reflect the close coupling between oxygen metabolism and glucose metabolism in the brain.

\subsection{Fluorescence imaging of cortical responses to forepaw stimulations}

First, whole bovine blood and 2-NBDG $(0.4 \mathrm{mM})$ were enclosed in $1 \mathrm{~mm}$ diameter glass tubes and imaged by our fluorescence setup [Figure 9a]. The fluorescence image shows that blood did not generate fluorescence while 2-NBDG did profusely.

Second, using the same experimental protocol as in PACT, we imaged the cortical responses to forepaw stimulations on a different mouse using the fluorescence setup. The results are shown in Figure 9b. While the white light image shows the detailed cortical vasculature, the fluorescence image shows the 2-NBDG distribution and thus the glucose metabolism. The RPS and LPS induced fluorescence intensity to increase by $5.1 \%$ and $6.2 \%$ in the SR of the contralateral hemisphere, respectively. Such increases indicate elevated glucose uptakes in the SR, which qualitatively agree with the glucose responses assessed by PACT.

\section{Discussion}

The pathways of oxygen metabolism and glucose metabolism are closely coupled in neuron activity. One of the recent uses of PACT is the label-free measurement of metabolic rate of oxygen $\left(\mathrm{MRO}_{2}\right)$ (Wang, 2008; Yao et al., 2011). Currently, except for blood flow speed, PACT can measure all the other parameters required for quantifying $\mathrm{MRO}_{2}$, namely vessel cross-section, oxygen saturation and total concentration of hemoglobin, and tissue volume. Recently, a few PA methods have been proposed for blood flow measurement in deep tissue, which may be applicable to PACT (Brunker and Beard, 2010; Fang et al., 2007; Sheinfeld and Eyal, 2012; Sheinfeld et al., 2010). Therefore, PACT has the potential to simultaneously image the two metabolic pathways: oxygen metabolism as described by others, and glucose metabolism as shown in this paper.

Previous studies by PET and fMRI have shown that forepaw stimulations can increase the cerebral metabolic rate of oxygen $\left(\mathrm{CMRO}_{2}\right)$ (Liu et al., 2004; Mandeville et al., 1998; Ogawa et al., 1994). Because the blood oxygenation change is much smaller than the blood flow change, such increase in $\mathrm{CMRO}_{2}$ is mainly attributed to the elevated blood perfusion (Liu et al., 2004; Mandeville et al., 1998; Ogawa et al., 1994). In our results, the intensified photoacoustic signal at $570 \mathrm{~nm}$ provided a direct evidence of the increase in blood perfusion. For absolute $\mathrm{CMRO}_{2}$ quantification, our future work will incorporate the oxygenation measurement by using multiwavelength measurements. 
In addition, we found that the glucose response area was confined within the SR, while the hemodynamic response area was much larger than that. Glucose response was a focal activity restricted to neurons responsible for the stimulated site. In contrast, hemodynamic response was less spatially restricted since fresh blood must be delivered from the major feeding arteries, through the capillaries of the SR, to the major draining veins. Moreover, the hemodynamic response may spread into neighboring vessels of the same vascular network.

Except for the dimensional discrepancy, the glucose response area was more homogenous than the hemodynamic response area. The latter was characterized by a strong vascular pattern. Moreover, there was a clear core for the glucose response, which is indicated by the double arrows in Figure 6a. Such a core was not seen in the hemodynamic response. This again suggests that glucose response was a focal activity. The core region contained the neurons most sensitive to the stimulated site.

In summary, using 2-NBDG as the exogenous contrast and hemoglobin as the endogenous contrast, we have demonstrated that PACT is capable of imaging the metabolic response of a mouse brain to forepaw stimulations. As a quantitative imaging modality, PACT can spectrally separate 2-NBDG and hemoglobin by virtue of its optical absorption contrast. As a fast imaging modality, PACT can acquire a volumetric image in less than two seconds with a laser repetition rate of $10 \mathrm{~Hz}$. This imaging speed can be further improved by a laser system with higher repetition rate and automatic wavelength switch. As a deep imaging modality, PACT can transdermally and transcranially localize the spatial patterns of the brain responses by virtue of its high ultrasonic resolution. With all these merits, we expect PACT to be applied to more brain metabolism studies in the future.

\section{Acknowledgments}

The authors appreciate Prof. James Ballard's close reading of the manuscript, and thank Arie Krumholz, Muhammad Chatni and Lidai Wang for useful discussion and technical assistance. This work was sponsored by National Institutes of Health (NIH) grants R01 EB000712, R01 EB008085, R01 CA134539, U54 CA136398, R01 EB010049, R01 CA157277, and R01 CA159959.

\section{References}

Azzi L, El-Alfy M, Martel C, Labrie F. Gender differences in mouse skin morphology and specific effects of sex steroids and dehydroepiandrosterone. Journal of Investigative Dermatology. 2005; 124:22-27. [PubMed: 15654949]

Bem M, Badea F, Draghici C, Caproiu MT, Vasilescu M, Voicescu M, Beteringhe A, Caragheorgheopol A, Maganu M, Constantinescu T, Balaban AT. Synthesis and properties of some new 4-amino-7 nitrobenzoxadiazole derivatives. ARKIVOC. 2007; 2007:87-104.

Brunker, J.; Beard, P. Pulsed photoacoustic Doppler flowmetry using a cross correlation method. Oraevsky, AA.; Wang, LV., editors. SPIE; San Francisco, California, USA: 2010. p. 756426-756428.

Carew, TJ. Behavioral neurobiology : the cellular organization of natural behavior. Sinauer Associates Publishers; Sunderland, Mass: 2000.

Cheng Z, Levi J, Xiong ZM, Gheysens O, Keren S, Chen XY, Gambhir SS. Near-infrared fluorescent deoxyglucose analogue for tumor optical imaging in cell culture and living mice. Bioconjugate Chemistry. 2006; 17:662-669. [PubMed: 16704203]

Fang H, Maslov K, Wang LV. Photoacoustic Doppler effect from flowing small light-absorbing particles. Phys Rev Lett. 2007; 99:184501. [PubMed: 17995411]

Fulham MJ, Bizzi A, Dietz MJ, Shih HHL, Raman R, Sobering GS, Frank JA, Dwyer AJ, Alger JR, Dichiro G. Mapping of Brain-Tumor Metabolites with Proton Mr Spectroscopic Imaging - Clinical Relevance. Radiology. 1992; 185:675-686. [PubMed: 1438744] 
Gamelin J, Maurudis A, Aguirre A, Huang F, Guo PY, Wang LV, Zhu Q. A real-time photoacoustic tomography system for small animals. Optics Express. 2009; 17:10489-10498. [PubMed: 19550444]

Gaudreault N, Scriven DRL, Laher I, Moore EDW. Subcellular characterization of glucose uptake in coronary endothelial cells. Microvascular Research. 2008; 75:73-82. [PubMed: 17531273]

Hu S, Maslov K, Wang LV. Second-generation optical-resolution photoacoustic microscopy with improved sensitivity and speed. Optics Letters. 2011; 36:1134-1136. [PubMed: 21479007]

Itoh Y, Abe T, Takaoka R, Tanahashi N. Fluorometric determination of glucose utilization in neurons in vitro and in vivo. Journal of Cerebral Blood Flow and Metabolism. 2004; 24:993-1003. [PubMed: 15356420]

Langsner RJ, Middleton LP, Sun J, Meric-Bernstam F, Hunt KK, Drezek RA, Yu TK. Wide-field imaging of fluorescent deoxy-glucose in ex vivo malignant and normal breast tissue. Biomedical Optics Express. 2011; 2:1514-1523. [PubMed: 21698015]

Li CH, Aguirre A, Gamelin J, Maurudis A, Zhu Q, Wang LV. Real-time photoacoustic tomography of cortical hemodynamics in small animals. Journal of Biomedical Optics. 2010; 15

Li ML, Oh JT, Xie XY, Ku G, Wang W, Li C, Lungu G, Stoica G, Wang LV. Simultaneous molecular and hypoxia imaging of brain tumors in vivo using spectroscopic photoacoustic tomography. Proceedings of the Ieee. 2008; 96:481-489.

Liu ZHM, Schmidt KF, Sicard KM, Duong TQ. Imaging oxygen consumption in forepaw somatosensory stimulation in rats under isoflurane anesthesia. Magnetic Resonance in Medicine. 2004; 52:277-285. [PubMed: 15282809]

Mandeville JB, Marota JJA, Kosofsky BE, Keltner JR, Weissleder R, Rosen BR, Weisskoff RM. Dynamic functional imaging of relative cerebral blood volume during rat forepaw stimulation. Magnetic Resonance in Medicine. 1998; 39:615-624. [PubMed: 9543424]

Maslov K, Zhang HF, Hu S, Wang LV. Optical-resolution photoacoustic microscopy for in vivo imaging of single capillaries. Optics Letters. 2008; 33:929-931. [PubMed: 18451942]

Mehagnoul-Schipper DJ, van der Kallen BFW, Colier WNJM, van der Sluijs MC, van Erning LJTO, Thijssen HOM, Oeseburg B, Hoefnagels WHL, Jansen RWMM. Simultaneous measurements of cerebral oxygenation changes during brain activation by near-infrared spectroscopy and functional magnetic resonance imaging in healthy young and elderly subjects. Human Brain Mapping. 2002; 16:14-23. [PubMed: 11870923]

Millon SR, Ostrander JH, Brown JQ, Raheja A, Seewaldt VL, Ramanujam N. Uptake of 2-NBDG as a method to monitor therapy response in breast cancer cell lines. Breast Cancer Research and Treatment. 2011; 126:55-62. [PubMed: 20390344]

Mink JW, Blumenschine RJ, Adams DB. Ratio of Central Nervous-System to Body Metabolism in Vertebrates - Its Constancy and Functional Basis. American Journal of Physiology. 1981; 241:R203-R212. [PubMed: 7282965]

Mosconi L, Mistur R, Switalski R, Tsui WH, Glodzik L, Li Y, Pirraglia E, De Santi S, Reisberg B, Wisniewski T, de Leon MJ. FDG-PET changes in brain glucose metabolism from normal cognition to pathologically verified Alzheimer's disease. European Journal of Nuclear Medicine and Molecular Imaging. 2009; 36:811-822. [PubMed: 19142633]

Natarajan A, Srienc F. Dynamics of glucose uptake by single Escherichia coli cells. Metab Eng. 1999; 1:320-333. [PubMed: 10937825]

Nieuwenhuys, R.; Donkelaar, H.J.t.; Nicholson, C.; Smeets, WJAJ.; Wicht, H. The central nervous system of vertebrates. Vol. Volume 1. Springer; Berlin ; New York: 1998.

O'Neil RG, Wu L, Mullani N. Uptake of a fluorescent deoxyglucose analog (2-NBDG) in tumor cells. Molecular Imaging and Biology. 2005; 7:388-392. [PubMed: 16284704]

Ogawa M, Magata Y, Ouchi Y, Fukuyama H, Yamauchi H, Kimura J, Yonekura Y, Konishi J. Scopolamine Abolishes Cerebral Blood-Flow Response to Somatosensory Stimulation in Anesthetized Cats - Pet Study. Brain Research. 1994; 650:249-252. [PubMed: 7953689]

Raichle ME, Gusnard DA. Appraising the brain's energy budget. Proceedings of the National Academy of Sciences of the United States of America. 2002; 99:10237-10239. [PubMed: 12149485] 
Schlemmer HPW, Pichler BJ, Schmand M, Burbar Z, Michel C, Ladebeck R, Jattke K, Townsend D, Nahmias C, Jacob PK, Heiss WD, Claussen CD. Simultaneous MR/PET imaging of the human brain: Feasibility study. Radiology. 2008; 248:1028-1035. [PubMed: 18710991]

Sheinfeld, A.; Eyal, A. Flow-dependant photothermal modulation of the photoacoustic response. Oraevsky, AA.; Wang, LV., editors. SPIE; San Francisco, California, USA: 2012. p. 8223-8221D.

Sheinfeld A, Gilead S, Eyal A. Photoacoustic Doppler measurement of flow using tone burst excitation. Optics Express. 2010; 18:4212-4221. [PubMed: 20389434]

Sheth RA, Josephson L, Mahmood U. Evaluation and clinically relevant applications of a fluorescent imaging analog to fluorodeoxyglucose positron emission tomography. Journal of Biomedical Optics. 2009; 14

Tsytsarev V, Maslov KI, Yao JJ, Parameswar AR, Demchenko AV, Wang LHV. In vivo imaging of epileptic activity using 2-NBDG, a fluorescent deoxyglucose analog. Journal of Neuroscience Methods. 2012; 203:136-140. [PubMed: 21939688]

Vishwanath K, Yuan H, Barry WT, Dewhirst MW, Ramanujam N. Using Optical Spectroscopy to Longitudinally Monitor Physiological Changes within Solid Tumors. Neoplasia. 2009; 11:889_ 900. [PubMed: 19724683]

Wang LV. Prospects of photoacoustic tomography. Medical Physics. 2008; 35:5758-5767. [PubMed: 19175133]

Wang LV. Multiscale photoacoustic microscopy and computed tomography. Nature Photonics. 2009a; 3:503-509. [PubMed: 20161535]

Wang, LV. Photoacoustic imaging and spectroscopy. CRC; Boca Raton: 2009b.

Wang, LV.; Wu, H.-i. Biomedical optics : principles and imaging. Wiley-Interscience; Hoboken, N.J.: 2007.

Wang XD, Pang YJ, Ku G, Xie XY, Stoica G, Wang LHV. Noninvasive laser-induced photoacoustic tomography for structural and functional in vivo imaging of the brain. Nature Biotechnology. 2003; 21:803-806.

Xia J, Guo ZJ, Maslov K, Aguirre A, Zhu Q, Percival C, Wang LHV. Three-dimensional photoacoustic tomography based on the focal-line concept. Journal of Biomedical Optics. 2011; 16

$\mathrm{Xu}$ MH, Wang LV. Universal back-projection algorithm for photoacoustic computed tomography (vol 71, art no 016706, 2005). Physical Review E. 2007; 75

Yao J, Maslov K, Hu S, Wang LV. Evans blue dye-enhanced capillary-resolution photoacoustic microscopy in vivo. Journal of Biomedical Optics. 2009; 14:054049. [PubMed: 19895150]

Yao J, Maslov KI, Shi Y, Taber LA, Wang LV. In vivo photoacoustic imaging of transverse blood flow by using Doppler broadening of bandwidth. Opt. Lett. 2010; 35:1419-1421. [PubMed: 20436589]

Yao J, Maslov KI, Zhang Y, Xia Y, Wang LV. Label-free oxygen-metabolic photoacoustic microscopy in vivo. Journal of Biomedical Optics. 2011; 16:076003. [PubMed: 21806264]

Yao J, Wang LV. Transverse flow imaging based on photoacoustic Doppler bandwidth broadening. Journal of Biomedical Optics. 2010; 15:021304. [PubMed: 20459226]

Yao J, Wang LV. Photoacoustic tomography: fundamentals, advances and prospects. Contrast Media Mol Imaging. 2011; 6:332-345. [PubMed: 22025335]

Zhang HF, Maslov K, Stoica G, Wang LV. Functional photoacoustic microscopy for high-resolution and noninvasive in vivo imaging. Nature Biotechnology. 2006; 24:848-851. 
a

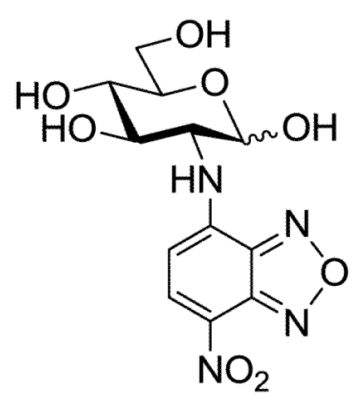

b

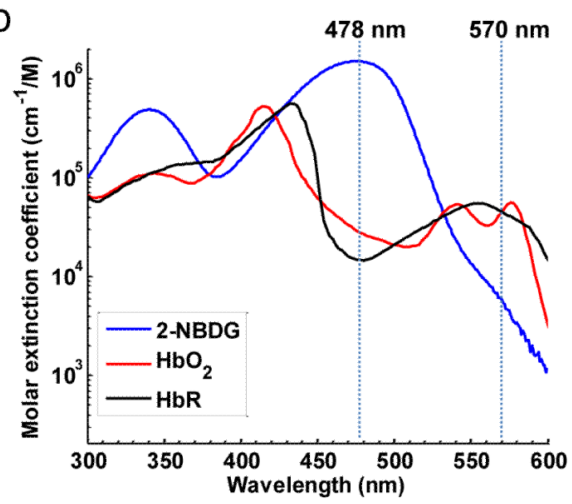

Figure 1.

2-NBDG $\left(\mathrm{C}_{12} \mathrm{H}_{14} \mathrm{~N}_{4} \mathrm{O}_{8}\right)$. (a) Chemical structure. (b) Molar extinction spectra of 2-NBDG, deoxy- and oxy-hemoglobin. 

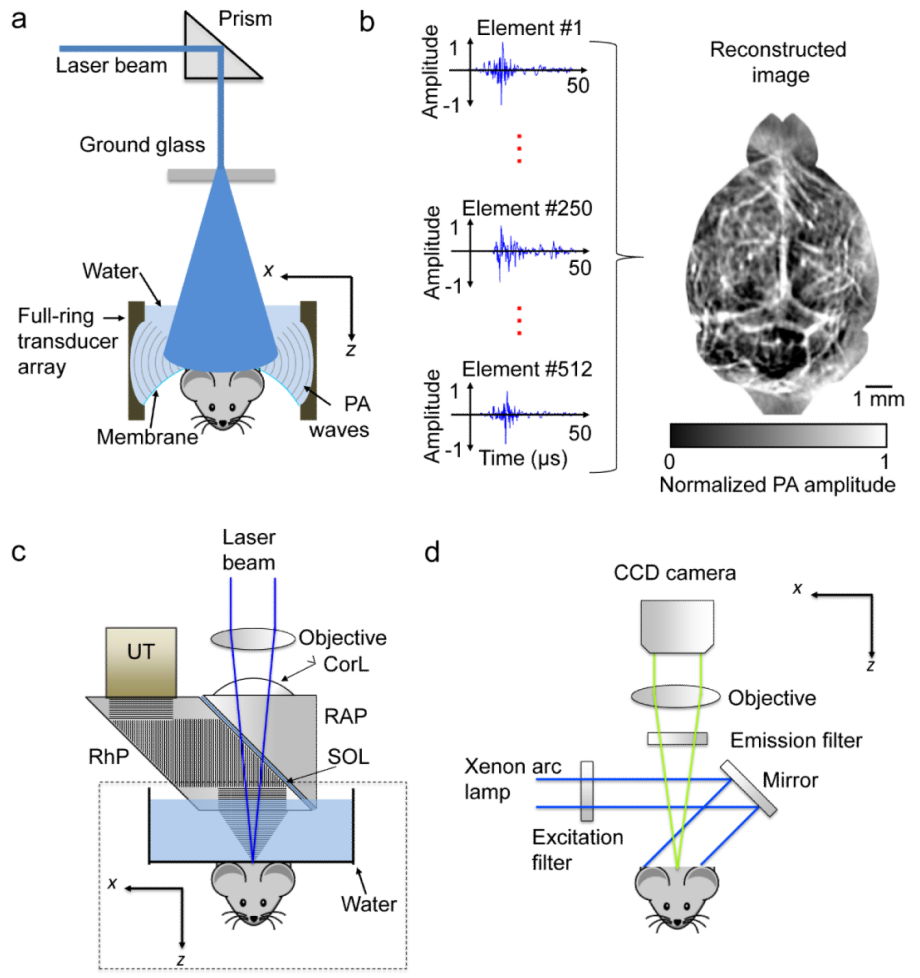

Figure 2.

Photoacoustic and fluorescence imaging systems. (a) Schematic of the PACT system. (b) In PACT, upon laser excitation, the time-domain PA signals recorded by each transducer element are back-projected into the 2D imaging space based on the delay times, and then summed to form an image of the mouse cortex. (c) Schematic of the OR-PAM system, where the capillary-level lateral resolution is provided by the tight optical focusing. UT, ultrasonic transducer; CorL, correction lens; RAP, right-angled prism; RhP, rhomboid prism; SOL, silicone oil layer. (d) Schematic of the fluorescence imaging system. 
a
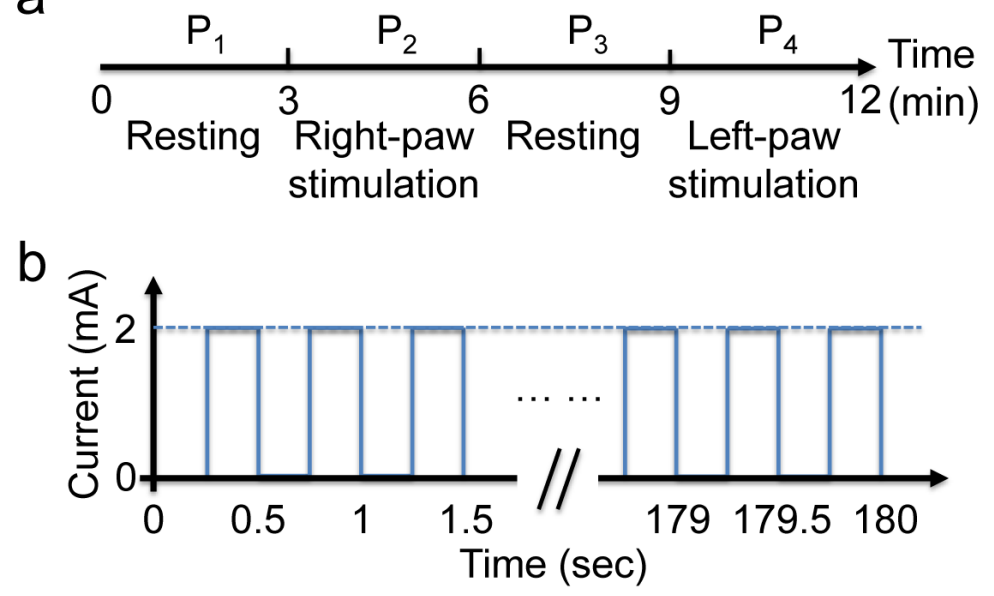

Figure 3.

Time course of forepaw stimulation. (a) At each wavelength, the procedure consisted of four periods: two resting periods $\left(\mathrm{P}_{1}\right.$ and $\left.\mathrm{P}_{3}\right)$ and two stimulation periods $\left(\mathrm{P}_{2}\right.$ and $\left.\mathrm{P}_{4}\right)$. Each period lasted for $3 \mathrm{~min}$. (b) Each stimulation period consisted of a train of electrical pulses with an amplitude of $2 \mathrm{~mA}$, a pulse width of $0.25 \mathrm{sec}$ and a repetition rate of $2 \mathrm{~Hz}$. 
a

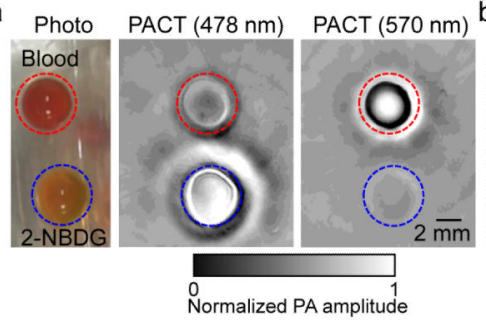

d

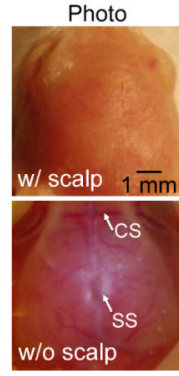

e

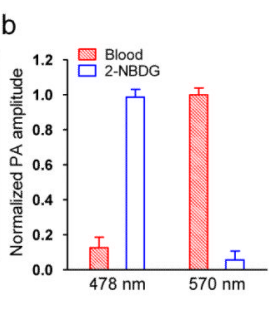

c 2-NBDG/Blood

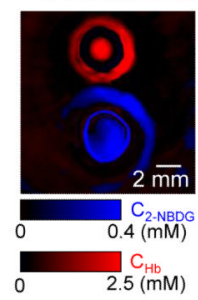

g 2-NBDG/Blood

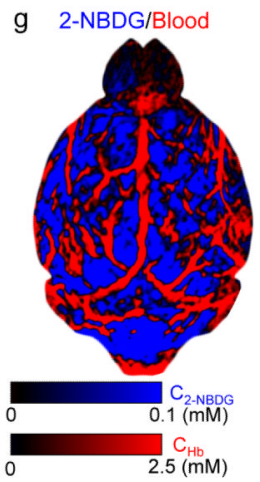

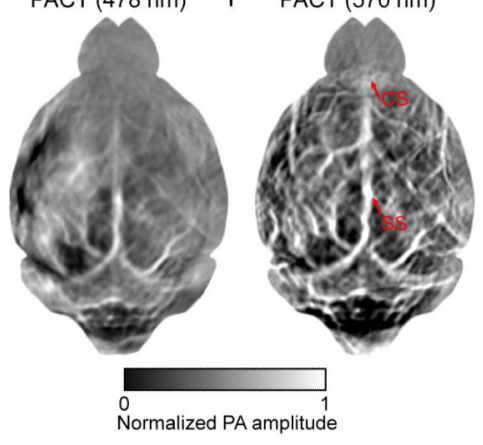

\section{Figure 4.}

Multi-wavelength PACT in vitro and in vivo. (a) Photograph (left) and PACT images (middle and right) of whole bovine blood and $0.4 \mathrm{mM} 2-\mathrm{NBDG}$ in a gelatin phantom. (b) Averaged PA amplitudes from blood and 2-NBDG, normalized by the PA amplitude of blood at $570 \mathrm{~nm}$. (c) Spectral separation of blood (shown in red) and 2-NBDG (shown in blue). $\mathrm{C}_{\mathrm{Hb}}$ : total hemoglobin concentration; C2-NBDG: 2-NBDG concentration. (d) Photograph of a mouse brain with its scalp intact (top) and removed (bottom). SS: sagittal sinus; CS coronal suture. (e-f) PACT images of the mouse brain at (e) 2-NBDG-dominant $478 \mathrm{~nm}$ and (f) hemoglobin-dominant $570 \mathrm{~nm}$, respectively. (g) Spectral separation of blood (shown in red) and 2-NBDG (shown in blue) in the brain. 

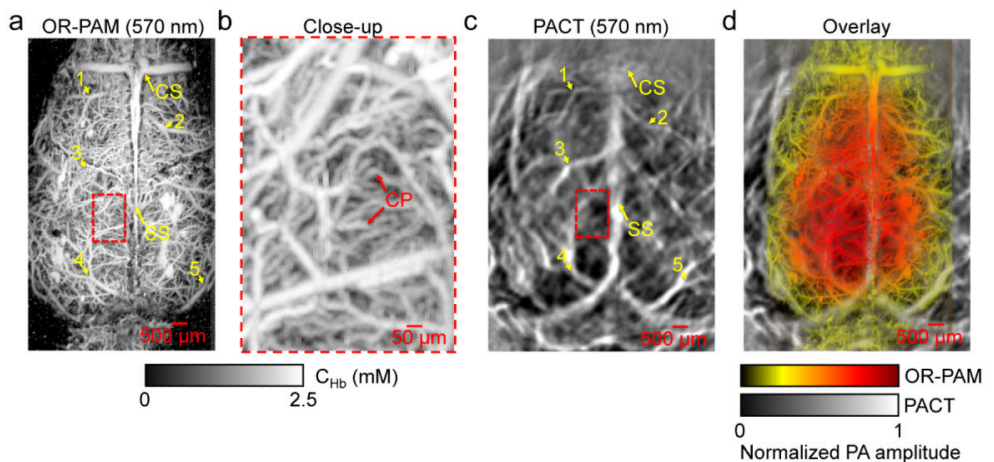

Figure 5.

High-resolution OR-PAM imaging of the mouse brain after PACT imaging. (a) OR-PAM image of the mouse brain, acquired at $570 \mathrm{~nm}$ with the scalp removed and the skull left intact. $\mathrm{C}_{\mathrm{Hb}}$ : total hemoglobin concentration; SS: sagittal sinus; $\mathrm{CS}$ : coronal suture. The numbers denote representative control points selected for image co-registration. (b) Close-up of region inside the dashed box in (a) showing the dense capillaries in the brain cortex. $\mathrm{CP}$ : capillary. (c) PACT image of the same mouse brain with intact scalp and skull. The numbers denote the control points corresponding to those in (a). (d) Co-registered OR-PAM image (shown in color) superimposed on the PACT image (shown in gray). 
a

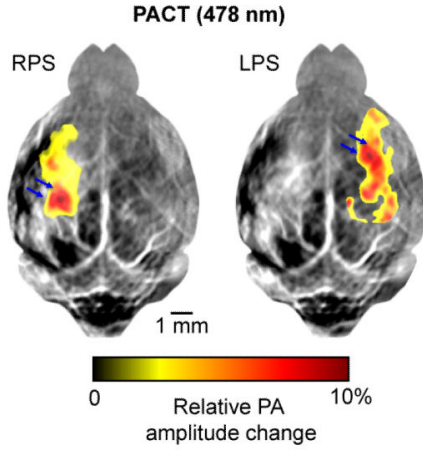

C

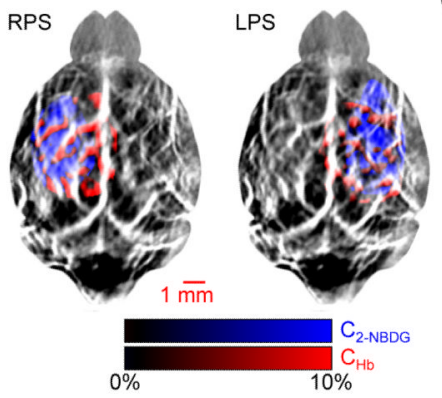

b

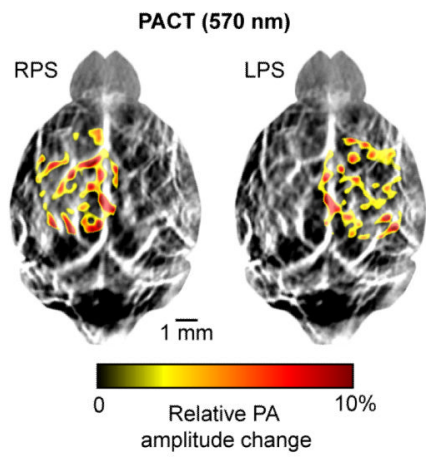

d

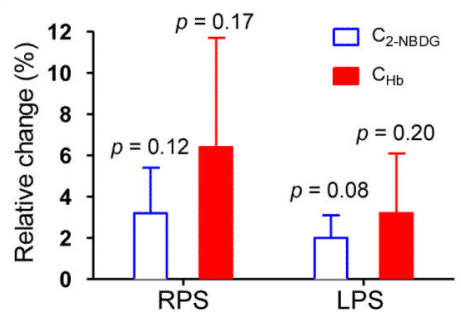

Figure 6.

PACT of the cortical responses to forepaw stimulations. (a-b) Relative changes of the PA amplitudes (shown in color) acquired at (a) 2-NBDG-dominant $478 \mathrm{~nm}$ and (b) hemoglobindominant $570 \mathrm{~nm}$ under the right paw stimulation (RPS) and left paw stimulation (LPS), superimposed on the resting-state image (shown in gray). The threshold for the overlay transparency was set to be $10 \%$ of the maximum response. (c) Overlaid images showing the relative changes of 2-NBDG concentration (C2-NBDG, shown in blue) and total hemoglobin concentration $\left(\mathrm{C}_{\mathrm{Hb}}\right.$, shown in red), superimposed on the resting-state image at $570 \mathrm{~nm}$ (shown in gray). (d) Relative changes of C2-NBDG and $\mathrm{C}_{\mathrm{Hb}}$ averaged over three mice. Error bars: standard deviation. The $p$ values were calculated from paired Student's ttest between stimulated states $\mathrm{P}_{2}$ or $\mathrm{P}_{4}$ and resting states $\mathrm{P}_{1}$ or $\mathrm{P}_{3}$. 
a

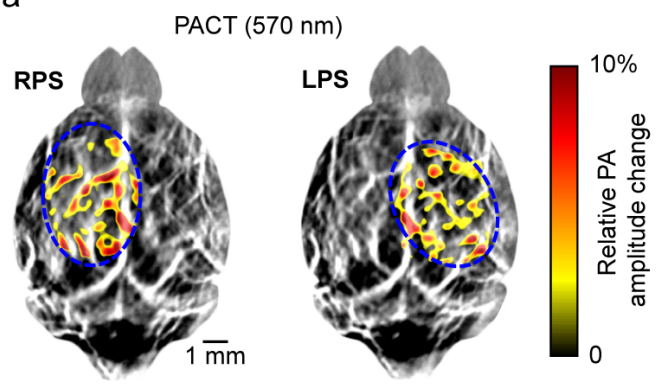

b

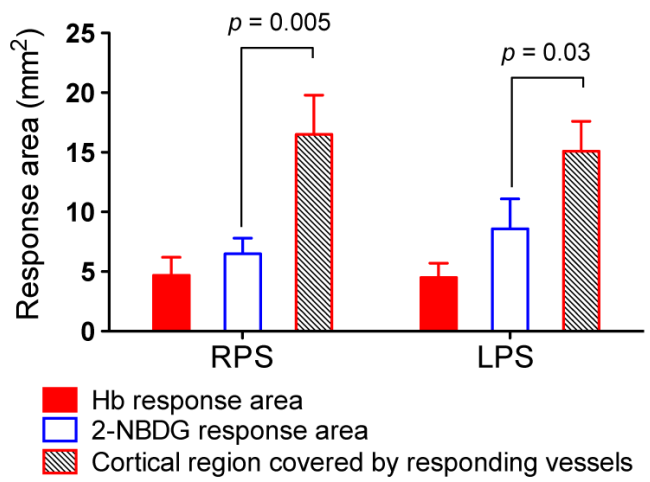

Figure 7.

Quantification of the cortical region covered by responding vessels. (a) An ellipse was used to fit the outline of the responding vessels, with the center, major axis, minor axis and orientation as the fitting variables. (b) Quantification of hemoglobin response area, 2-NBDG response area and cortical region covered by responding vessels, averaged over three mice. Error bars: standard deviation. The $p$ values were calculated from paired Student's t-test between the 2-NBDG response area and the cortical region covered by responding vessels. 

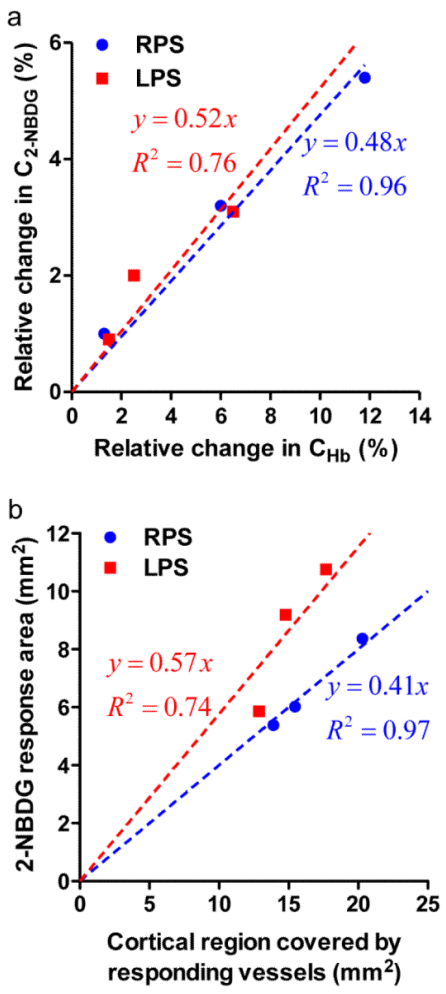

Figure 8.

Correlation of the hemodynamic response and glucose response. (a) Linear regression of the 2-NBDG response amplitude and the hemoglobin response amplitude for RPS and LPS on three mice. (b) Linear regression of the 2-NBDG response area and cortical area covered by responding vessels for RPS and LPS on three mice. 

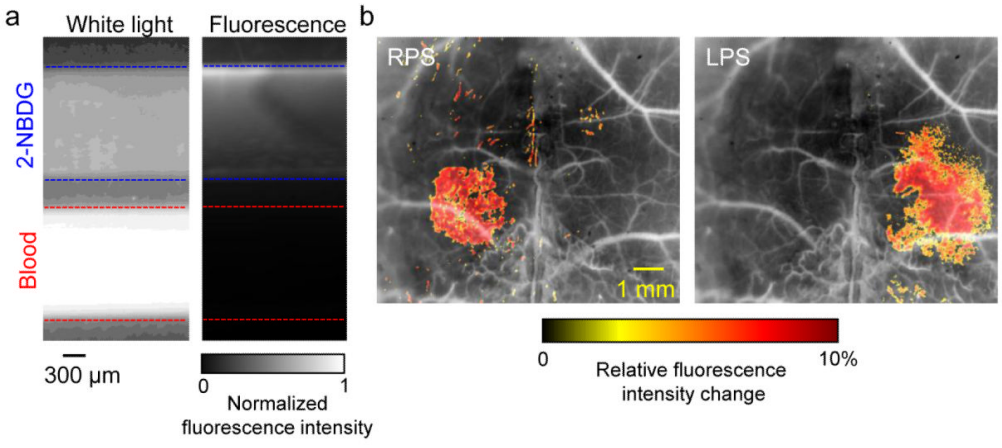

Figure 9.

Fluorescence imaging of mouse brain responses to forepaw stimulations. (a) Left: intensityreversed white-light image of two glass tubes filled with 2-NBDG $(0.4 \mathrm{mM})$ and whole bovine blood. Right: fluorescence image of the two tubes. (b) Relative changes of the fluorescence intensity under RPS and LPS (shown in color), superimposed on the intensityreversed white-light image of the brain cortex (shown in gray), with the scalp removed and skull left intact. The threshold for the overlay transparency was set to be $10 \%$ of the maximum response. 